\title{
An Agent-based Model to Study Effects of Team Processes on Compliance with Safety Regulations at an Airline Ground Service Organization
}

\author{
Alexei Sharpanskykh and Rob Haest \\ Delft University of Technology, Faculty of Aerospace Engineering \\ Kluyverweg 1, 2629 HS Delft, The Netherlands \\ \{o.a.sharpanskykh, r.c.s.haest\}@tudelft.nl
}

\begin{abstract}
Maintaining high levels of safety under conditions of ever increasing air traffic is a challenging task. Failures to comply with safety-related regulations are often considered to be important contributors to safety occurrences. To address the issue of compliance, approaches based on external regulation of the employees' behavior were proposed. Unfortunately, an externally imposed control is often not internalized by employees and has a short-term effect on their performance. To achieve a long-term effect, employees need to be internally motivated to adhere to regulations. Theories from social sciences propose that team processes play an important role in the dynamics of individual motivation. In this paper an agent-based model is proposed, by which the impact of social interaction and coordination in teams of platform employees on their individual motivation and compliance with safety regulations at an airline ground service organization are explored. The model was simulated and partially validated by a case study performed at a real airline ground service organization. The model was able to reproduce behavioral patterns of compliance of the platform employees in this study.
\end{abstract}

Keywords: compliance, agent-based model, motivation, cognitive models, social contagion.

\section{Introduction}

The amount of air traffic increases with every passing year. The performance pressures imposed nowadays on the actors in air transportation make it difficult to achieve safety targets formulated by regulatory organizations. According to the aviation statistics [2], most of the safety occurrences happen not during the flight, but on the ground, e.g., during aircraft ground handling operations and aircraft maintenance operations. Decreasing the number of ground safety occurrences has a high priority in many airlines in different countries. To achieve this aim some airlines use Ramp Line Operations Safety Assessments (LOSA) [2] - a monitoring tool for measuring and identifying the adherence to safety regulations on the platform. Unfortunately, the introduction of ramp LOSA in the ground service organization under study did not result in a decrease in the number of ground safety occurrences. 
To achieve a long-term compliance, employees need to be internally motivated to adhere to regulations [3]. In this paper, based on a theoretical fundament from social sciences we build an agent-based model to explore the role of team processes in individual motivation and compliance of platform employees with safety regulations in an airline ground service organization. In particular we consider a specific task of the aircraft arrival procedure - Foreign Object Damage (FOD) check. Foreign object is any object that should not be located near aircraft as it can damage aircraft or injure personnel. According to [1], the improper execution of FOD checks costs airlines and airports millions of dollars every year. Nevertheless, the ramp LOSA statistics showed that FOD checks are often not performed by platform employees.

The proposed model elaborates the motivation and decision making of the platform employees whether or not to perform the FOD check. In this elaboration, next to social also cognitive and organizational factors are taken into account. Furthermore, the model includes individual and social learning of agents representing the employees and addresses two modes of reasoning of agents - explicit rational and implicit automatic (habits). At the social level we are particularly interested in how coordination influences the motivation and compliance with safety regulations of the agents. In the organization under study, first implicit coordination was used in the teams, when the FOD check task was not explicitly allocated to any team member, but to the team as a whole, and could be executed by any team member who decides to do so. After some time, a new arrival procedure was introduced, which used explicit coordination. In this case, the FOD check task was explicitly assigned to a specific team member. We identified by simulation that in the case of implicit coordination a high level of compliance was maintained in the presence of a high managerial control and the level of compliance dropped quickly when the control decreased to a lower level. On the contrary, in the case of explicit coordination the compliance did not decrease significantly in the presence of low control. These simulation outcomes are well supported by the ramp LOSA statistics from the real ground service organization.

The paper is organized as follows. In the following Section 2 the theoretical basis of the model is described. In Section 3 the proposed agent-based model is provided. Main results from the simulation study are discussed in Section 4. The paper ends with conclusions and discussions.

\section{Theoretical Background}

The theoretical basis of the model comprises several theories from social sciences described below. These theories address human needs, the way how humans reason about their needs and make choices to act based on this reasoning. All the theories used for the model development have a good empirical support.

Self-determination theory [3] is a theory of human motivation, which addresses people's universal, innate psychological needs and tendencies for growth and fulfillment. Specifically, the theory postulates three types of basic needs:

- the need for competence concerns the people's inherent desire to be effective in dealing with the environment; 
- $\quad$ the need for relatedness concerns the universal disposition to interact with, be connected to, and experience caring for other people;

- the need for autonomy concerns people's universal urge to be causal agents, to experience volition.

In line with other motivation theories [6, 7], in addition to the needs listed above, the need for safety was added, which is particularly relevant for the ground service organization, in which physical injuries are not uncommon.

Based in needs individual goals can be defined. Higher level individual goals may be refined in goal hierarchies as described in [8]. To achieve or maintain his or her goals, an individual considers different behavioral options (actions or plans). One of the theories that explains why individuals choose one option over another is the Expectancy Theory of Motivation by Vroom [7]. According to the theory, when an individual evaluates alternative possibilities to act, he or she explicitly or implicitly makes estimations for the following factors: expectancy, instrumentality and valence.

Expectancy refers to the individual's belief about the likelihood that a particular act will be followed by a particular outcome (called a first-level outcome). Its value varies between 0 and 1 .

Instrumentality is a belief concerning the likelihood of a first level outcome resulting into a particular second level outcome; its value varies between -1 and +1 . A second level outcome represents a desired (or avoided) state of affairs that is reflected in the agent's goals.

Valence refers to the strength of the individual's desire for an outcome or state of affairs; it is also an indication of the priority of goals.

Values of expectancies, instrumentalities and valences may change over time, in particular due to individual and social learning. The motivational force of an individual $i$ to choose option to act $k$ is calculated as:

$$
F_{k, i}(t)=\sum_{l=1}^{n} E_{k l, i}(t) \sum_{h=1}^{m} V_{h, i}(t) I_{k l h, i}(t)
$$

Here $E_{k l, i}(t)$ is the strength of the expectancy that option $k$ will be followed by outcome $l ; V_{h, i}(t)$ is the valence of the second level outcome (a goal) $h ; I_{k l h, i}(t)$ is perceived instrumentality of outcome $l$ for the attainment of outcome $h$ for option $k$.

The Vroom's theory describes the process of rational decision making. However, repetitive actions such as occur during aircraft handling may over time become automatic, i.e., a habit. The dual process theory [5] distinguishes System 1 and System 2 thinking. While System 2 is used for rational, rule-based and analytic thinking, System 1 is associated with unconscious, implicit and automatic reasoning. Depending on the dynamics of environmental changes, an individual switches between the systems. Both systems are used in the model and the case study considered in the paper.

\section{The Agent-based Model}

To develop the model, an extensive one year study was performed at a real ground service organization. The data were gathered by observation, questionnaires and 
interviews with employees playing different roles in the organization. The collected data were separated in two data sets. The first set contained data on the organizational context (i.e., formal organizational structures and processes, norms and regulations) and on local processes and characteristics of the organizational agents. This dataset was used for the model initialization. To represent the uncertainty and variability of the components of the model, most of the parameters were specified by intervals with a uniform distribution. The second set contained data describing global organizational or systemic properties (such as ramp LOSA statistics), which were used for the model validation. Because of the space limitations, only a part of the model is described below. For the complete model description please refer to [9].

\section{Specification of decision making of agents}

Decision making by the Platform Employee agents whether or not to perform FOD check was modeled by using the Vroom's expectancy theory (Fig. 1). To initialize the expectancies, instrumentalities and valences of the model for each agent three classes of values were introduced: Low, Medium and High. Most of the numerical scales of these parameters were divided equally among the classes: Low for [0, 0.33), Medium for $[0.33,0.67)$, and High for $[0.67,1]$.

The expectancy theory model was used for System 2 reasoning. When the same operations were routinely executed by a Platform Employee agent, the agent's System 2 reasoning was gradually shifting to System 1 reasoning - a habit had been formed. This shift was modeled by the dynamics of agent's $i$ openness parameter $\alpha_{i}$ :

$$
\alpha_{i}(t+\Delta t)=\alpha_{i}(t)+\zeta\left(\alpha_{i}^{\min }-\alpha_{i}(t)\right) \Delta t,
$$

where $\alpha_{i}^{\mathrm{min}}$ is the minimum perceptive openness of agent $i$ (set to 0.1 in the simulation), $\zeta$ is the rate of transition from System 2 to System 1 . It depends on the execution frequency of the operation by the agent, as well as on the agent's personal characteristics. In the simulation $\zeta=0.015$, meaning that it takes around two months to form a new habit.

When procedural rules change, an agent needs to adapt to a new situation and reconsider options by switching from System 1 to System 2: the agent $i$ 's openness is set to its initial value $\alpha_{i}(0)$ and the process of the new habit formation starts again.

A similar expectancy theory model was created for option 2 - 'Not to perform FOD check'. It has the same types of parameters, but their values are different.

In the simulation, every time when an agent $i$ considers explicitly (System 2) or implicitly (System 1) whether or not to perform FOD check, motivation forces $F_{1, i}$ and $F_{2, i}$ for both options are calculated by (1). Then, the agent performs FOD check with probability $\left(F_{\max }+F_{1, i}\right) /\left(2 F_{\max }+F_{1, i}+F_{2, i}\right)$. The normalization with $F_{\max }$ is used to compensate for the negative values of the instrumentalities.

\section{Specification of agent learning and social interaction}

Two types of learning were modeled: individual and social learning of agents.

An agent learns individually by observing a feedback from the environment on its action. In the decision making model from Fig. 1, the individual learning was realized by updating values of expectancies (E) based on the following observations: 


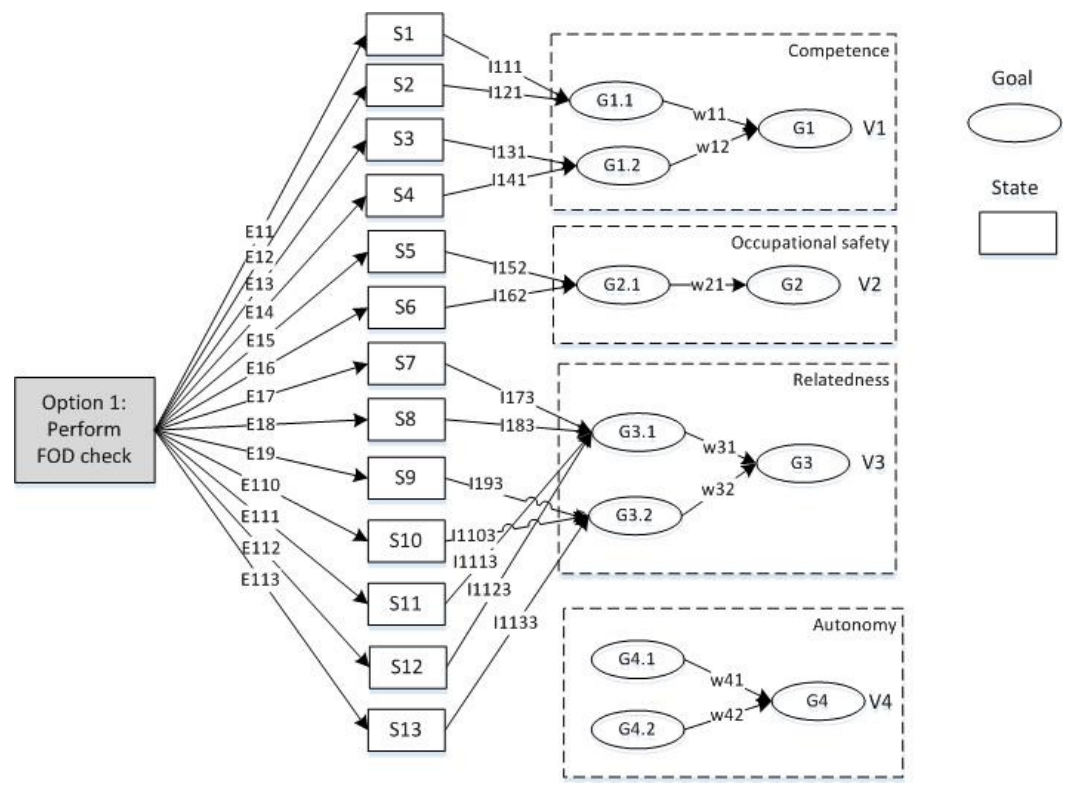

Fig. 1. Decision making model of a Platform Employee agent for performing FOD check based on the Expectancy Theory with expectancies (E), instrumentalities (I), states (S) and valences $(\mathrm{V})$. For readability purposes the time parameter and agent indexes were omitted.

Table 1. The goals and states of the decision making model provided in Fig.1.

\begin{tabular}{|c|c|}
\hline Goals & States \\
\hline Achieve a high level of competence & S1 Action saves time \\
\hline G1.1 Achieve the highest time efficiency & S2 Action costs additional time \\
\hline $\begin{array}{l}\text { G1.2 Prevent aircraft, equipment and/or } \\
\text { infrastructural damage }\end{array}$ & $\begin{array}{l}\text { S3 Action results in aircraft, equipment or } \\
\text { infrastructural damage } \\
\text { S4 Action prevents aircraft, equipment or } \\
\text { infrastructural damage }\end{array}$ \\
\hline $\begin{array}{l}\text { G2 Achieve a high level of occupational } \\
\text { safety }\end{array}$ & $\begin{array}{l}\text { S5 Action results in personal injury } \\
\text { S6 Action prevents personal injury }\end{array}$ \\
\hline G2.1 Prevent personal injury & $\begin{array}{l}\text { S7 Action is in alignment with the team } \\
\text { member norms }\end{array}$ \\
\hline $\begin{array}{l}\text { G3 Maintain sense of belonging and } \\
\text { attachment to colleagues }\end{array}$ & $\begin{array}{l}\text { S8 Action is not in alignment with the team } \\
\text { norms }\end{array}$ \\
\hline G3.1 Maintain high team acceptance & S9 Action is in alignment with sector \\
\hline G3.2 Maintain high management acceptance & management norms \\
\hline & $\begin{array}{l}\text { S10 Action is not in alignment with sector } \\
\text { management norms }\end{array}$ \\
\hline $\begin{array}{l}\text { G4 Achieve a high control over own } \\
\text { behavior and goals }\end{array}$ & $\begin{array}{l}\text { S11 Reprimand received from team member } \\
\text { S12 Renrimand received from team leader }\end{array}$ \\
\hline $\begin{array}{l}\text { G4.1 Achieve a high level of freedom in the } \\
\text { execution of tasks }\end{array}$ & S13 Reprimand received from sector \\
\hline $\begin{array}{l}\text { G4.2 Achieve high psychological ownership } \\
\text { of rules }\end{array}$ & \\
\hline
\end{tabular}


- An agent observes whether or not a reprimand from other agents is provided, when the agent does not comply with regulations (E111, E112, E113).

- After the successful execution of a task, an agent observes how much time it took and how it influenced the execution time of the operation (E11, E12).

- When an agent does not perform a FOD check, a safety occurrence could occur. The agent is able to observe such occurrences (E13, E14).

Furthermore, the Platform Employee agents are able to observe the execution of operations by other agents in their teams and to learn from these agents by verbal communication. Social learning is modeled as the process of social contagion [4]. By this process expectancies $E_{k l, i}(t)$ were updated as:

$$
E_{k l, i}(t+\Delta t)=E_{k l, i}(t)+\delta_{k l, i}(t) \Delta t
$$

Here $\delta_{k l, i}(t)=\sum_{j \in T} \gamma_{j, i}(t)\left(E_{k l, j}(t)-E_{k l, i}(t)\right) / \sum_{j \in T} \gamma_{j, i}(t)$ is the amount of change of the agent $i$ 's state; $T$ is the set of the agents in the team. A weight $\gamma_{j, i} \in[0,1]$ is the degree of influence of agent $j$ on agent $i$ defined as:

$$
\gamma_{j, i}(t)=\alpha_{i}(t) \varepsilon_{j}(t) \beta_{j i}
$$

$\alpha_{i}(t)$ and $\varepsilon_{j}(t)$ are the agent characteristics - the openness of information recipient agent $i$ and the expressiveness of information provider agent $j$, and $\beta \in[0,1]$ is the strength of the information channel between the two agents.

Identification of shared beliefs, norms and values of (groups of) agents

By field observations and interviews a team norm was identified. The norm applies to situations in which a team arrives too late at an aircraft stand while the aircraft is waiting for the docking process. To save time, the FOD check is omitted and the arrival procedure starts directly. Field data revealed that employees who execute the check in the described situation get a social reprehension from other team members. This influences the achievement of goal G3.1, which is driven by the alignment of the decision option with the team norms and team leader norms.

The Team Leader agent's and Sector Manager agent's norms are in line with the organizational regulations.

\section{Specification of coordination mechanisms in teams of agents}

Before the introduction of the new arrival procedure the tasks were coordinated implicitly, in an ad-hoc manner. In particular, the FOD check task was executed by different members, who had decided to perform it or was not executed at all. This way of working is described by the model provided above.

After the introduction of the new procedure each agent in the team was assigned a specific task. Field study data indicated that the probability for team member reprimands and team leader reprimands have increased significantly due to the explicit task division. Thus, in the decision making model of the agent responsible for the FOD check the relevant parameters were changed for the new procedure.

\section{Simulation study}

We consider a real scenario, which occurred in the past and consists of five periods: 
Before the introduction of the new arrival procedure, when implicit coordination was used in the teams:

P1: the first period with a limited managerial control over the execution of the platform operations and limited safety information provision.

P2: the second period ( 8 weeks) with a high managerial control after many safety occurrences happened in the first period.

P3: the third period in which the release of managerial control occurs over time.

Transition to the new arrival procedure, which includes explicit coordination:

P4: in the fourth period information sessions were organized to explain the purpose and effects of the procedural change to the employees. High managerial control was applied in the first months after the introduction of the new procedure.

P5: in the fifth period after one month after the introduction of the new procedure the intensity of information provision and the managerial control decreased.

A team consists of 5 agents: a team leader and 4 platform employees. In the simulation the agents in the teams communicate with each other in a random order.

In line with the empirical findings, two types of agents in the teams are modeled: more expressive agents with $\varepsilon_{i} \in[0.5,0.9]$ and less expressive agents with $\varepsilon_{i} \in[0.1$, $0.5]$. Each agent can be of either type with an equal probability. The openness of an agent $\alpha_{i}$ is assigned a wide range [0.1,0.9] to represent the diversity of agents. In each simulation run the agents' parameters are randomly instantiated from the uniformly distributed intervals introduced above.

In the simulation, one simulation day is divided in three shifts. During normal operations, on average, the arrival procedure is executed three times at each shift. The simulated time period is 200 working days.

In the following we discuss the dynamics of the motivational forces obtained in the simulation. In period $\mathrm{P} 1$, after the initialization phase, most of the agents have a relatively constant motivational force for both decision options. The motivational forces to perform FOD checks are low as the organization neither sufficiently controls the execution of operations nor creates a sufficient awareness about the importance of FOD checks. Some agents in the team even prefer not to perform the check. By the end of the phase the agents function in System 1 mode of reasoning. In the beginning of P2 the organization introduces more frequent managerial control and reprimands. To adapt to the new circumstances, the agents switch to System 2 mode of reasoning. Such a change results in an increased motivation to perform FOD checks and a decreased motivation not to do so of all agents in all teams. The differences in motivation are explained by differences in the individual characteristics of the agents. However, when after 8 weeks the control and information provision is gradually removed, the agents start gradually returning to their previous state. This form of motivated behavior is known in the literature as externally regulated behavior [3]. These behavioral patterns were also observed in the organization under study.

The introduction of the new procedure with the explicit coordination results in $\mathrm{P} 4$ in a higher motivation levels to perform FOD check and a lower motivation levels not to perform the check than in the previous three phases (Fig.2). Thus, the decision options have become better discriminated than in the previous periods. In P5 the managerial control is gradually removed. However, the motivational forces not to 
perform FOD check do not increase significantly. This effect can partly be explained by a better information provision and the social control.
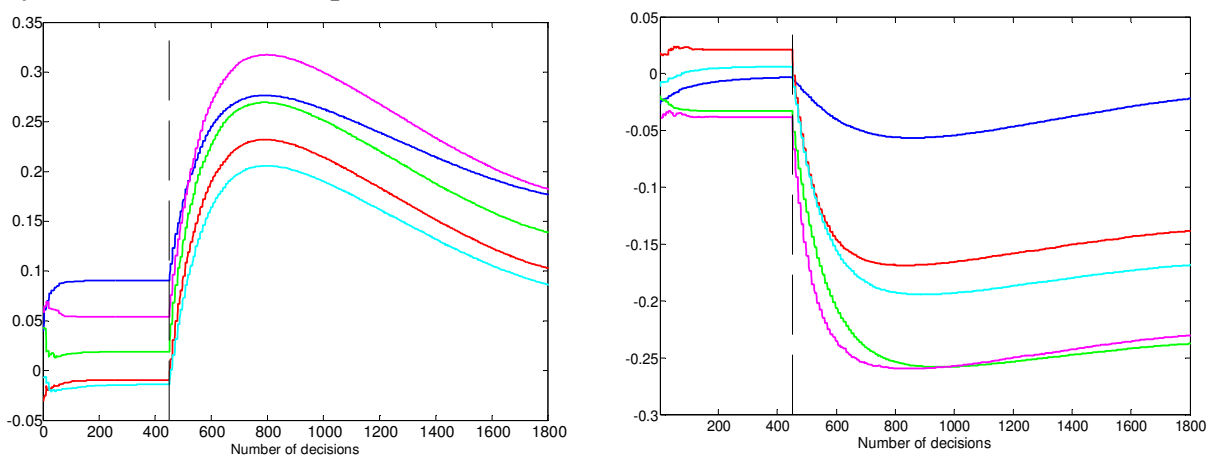

Fig. 2. The motivational forces of the agents in a team to perform FOD check (left) and to not perform FOD check (right) in the periods P4 and P5 with the explicit coordination in the teams. The dashed vertical line indicates the beginning of fourth period (increased control).

The model simulation outcomes were also compared to the ramp LOSA statistics of the FOD checks execution in the ground service organization. The model was able to capture the trends in the real ramp LOSA data. The Student's two-sample t-test performed on real and simulated data for periods P1-P3 supported the null-hypothesis at the significance level 5\%. The statistics about periods P4 and P5 is currently insufficient to draw substantiated conclusions. However the gathered data indicated that the compliance has increased substantially, which is in line with the simulation model prediction. The model predicts that the compliance with safety regulations will not drop as much as in P3 after the managerial control is decreased in P5. Thus, according to the model, the agents will not return to the safety-compromising habits.

\section{Discussion and Conclusions}

In this paper a formal agent-based motivation model is presented, based on an integrated theoretical basis from social sciences. All the theories used for the model development were extensively validated by experiments with human subjects.

In the study presented in the paper a good agreement is demonstrated between the simulated data obtained using the proposed model and the real data from the ground service organization under study. Based on the developed model, global sensitivity analysis was performed to identify parameters with the highest impact on the compliance both in the implicit and explicit coordination case. This analysis indicated that the parameters that define social relations and processes in teams are of the utmost importance in the case of explicit coordination. More detailed results on the sensitivity analysis are presented in [9].

To the best knowledge of the authors, it is a first attempt to approach the problem of compliance in airline ground service organizations by a model-driven simulation study of the employees' motivation. 


\section{References}

1. Boeing. (1998). Foreign object debris and damage prevention. Retrieved from http://www.boeing.com/commercial/aeromagazine/aero_01/textonly/s01txt.html

2. de Boer, R. J., Koncak, B., Habekotté, R., \& van Hilten, G. J. (2011). Introduction of rampLOSA at KLM Ground Services. In Human Factors of Systems and Technology, Human Factors and Ergonomics Society Annual Meeting. Maastricht: Shaker Publishing.

3. Deci, E. L., \& Vansteenkiste, M. (2004). Self-determination theory and basic need satisfaction: Understanding human development in positive psychology. Ricerche di Psichologia, 27, pp.17-34.

4. Hegselmann R., Krause, U. Opinion dynamics and bounded confidence: models, analysis and simulation, J.of Artificial Societies and Social Simulation 5, 3 (2002)

5. Kahneman, D. (2011). Thinking, fast and slow. New York: Farrar, Straus and Giroux.

6. McClelland, D. C. (1987). Human motivation. CUP Archive.

7. Pinder, C.C. Work motivation in organizational behavior. NJ: Prentice-Hall (1998)

8. Popova, V., Sharpanskykh, A. (2011). Formal Modelling of Organisational Goals Based on Performance Indicators. Data \& Knowledge Engineering, Vol. 70, Issue 4, pp. 335-364

9. Sharpanskykh, A., Haest, R. An Agent-based Model to Study Effects of Team Processes on Compliance with Safety Regulations at an Airline Ground Service Organization. Technical Report, TU Delft (2015)

(www: http://homepage.tudelft.nl/j11q3/papers/tech_report_182014.pdf) 